\title{
A NOTE ON TAUBERIAN OPERATORS
}

\author{
JESÚS ARAUJO \\ Departamento de Matemáticas \\ ETSI Industriales \\ Universidad de Oviedo \\ Castiello de Bernueces \\ 33204 Gijón, Spa1n

\section{J. MARTINEZ-MAURICA} \\ Departamento de Matemáticas \\ Facultad de Clencias \\ Unfiversidad de Santander \\ Av. Los Castros \\ 39071 Santander, Spain
}

(Recelved May 20, 1988 and in revised form August 23, 1988)

ABSTRACT. In this note we prove the existence of operators which are not Tauberian even though they satisfy properties about restrictions being Tauberian. The operators are defined on Banach spaces which contain a somewhat reflexive, non-reflexive subspace. This gives an answer to a question proposed by R. Neidinger [1].

KEY WORDS AND PHARSES. Tauberian Operators, and Semi-Fredholm Operators. 1980 AMS SUBJECT CLASSIFICATION CODES. 47 A56.

1. INTRODUCTION.

Throughout this note $E, F$ are infinite-dimensional Banach spaces over the real or complex fleld. All operators $\mathrm{T}: \mathrm{E} \longrightarrow \mathrm{F}$ are assumed to be linear and continuous. Given $T \in L(E, F)$ the notation $T \mid Z$ denotes the restriction of $T$ to the subspace $Z$ of $E$.

Recall that an operator $T \in L(E, F)$ is said to be semf-Fredholm if its null space $N(T)$, 18 finite-dimensional and its range space $R(T)$ is closed. Also, a Tauberian operator, as defined by D. Garling and A. Wilansky in [2], is a bounded linear operator $T \in L(E, F)$ such that $T$ " preserves the natural embedding of $E$ into its double dua 1, 1.e., T"x" $\in$ F Implies $x^{\prime \prime} \in$ E. Some relationships between these two classes of operators have been studied in [1], [3], [4] and [5]. In particular, if $R(T)$ is closed, then $T$ is Tauberian if only if $N(T)$ is reflexive.

It is we11-known that the restriction of a semi-Fredholm operator to any closed subspace is again a semi-Fredholm operator. In the opposite direction it is 
worthwhile to mention the following result that is basically due to $\mathrm{T}$. Kato [6],

THEOREM 1 (c.f. [6]). Let $E, F$ be Infinite-dimensional Banach spaces. Assume that $\mathrm{T}: \mathrm{E} \longrightarrow \mathrm{F}$ is an operator such that every infinite-dimensional closed subspace $Z$ of $\mathrm{E}$ contains an infintie-dimensional closed subspace $W$ for which $T / W$ is semiFredholm. Then $T$ is semi-Fredholm.

It follows that in order to see that a given operator $T$ is semi-Fredholm, it is enough to assure that its restriction to every closed subspace with a Schauder basis Is semi-Fredholm.

Another related result is the following theorem due to $R$. Neidinger in which Banach spaces with no infinite-dimensional reflexive subspace are called "purely nonreflexive" spaces.

THEOREM 2 ([1], p. 26). Let $E$ be a weakly sequentially complete Banach space and let $T \in L(E, F)$. Then $T$ is Tauberian $1 f$ (and only if) $T \mid Z$ is semi-Fredholm for all purely non-reflexive closed subspaces $Z$ of $E$.

In view of the preceding theorem, $R$. Neldinger raised the following question ([1], p. 139): If $T \in L(E, F)$, restricted to any purely non-reflexive closed subspace is semi-Fredholm, is $T$ Tauberian?. Indeed, the answer is positive if $E$ is reflexive. Then, we assume that $E$ is not reflexive. In this case there are some trivial situations for which the answer is negative (e.g., let $E$ be a somewhat reflexive space, that is, every infinite-dimensional subspace of $E$ contains an infinite-dimensional reflexive subspace, and let $T$ be a finite rank operator). Our next example gives a negative answer to the question raised by $R$. Neidinger in a nontrivial situation.

EXAMPL. Let $J$ be the James space and 1 et $T: J \times 1^{1} \longrightarrow 1^{1}$ be the operator defined by $T(x, y)=y$.

Since $R(T)$ is closed and $N(T)=J$ is not reflexive then, $T$ is not Tauberian. Now, let $Z$ be a purely non-reflexive closed subspace of $J \times 1^{1}$. Since $J$ is somewhat reflexive, $N(T \mid Z)=N(T) \cap Z$ is finite-dimensional; otherwise, $N(T \mid Z)$ would contain an infinite-dimensional reflexive subspace, which contradicts our assumption over $Z$.

Also, $N(T)$ and $Z$ are totally Incomparable Banach spaces (1.e., there exists no infinite-dimensional Banach space which is isomorphic to a subspace of $N(T)$ and to a subspace of $\mathrm{Z}$ ). This implies that $N(T)+Z$ is closed in $J \times 1^{1}$ [7] and hence, $T(Z)=R(T \mid Z)$ is closed by the open mapping theorem.

Thus, $T \mid Z$ is semi-Fredholm for all purely non-reflexive closed subspaces.

\section{MAIN RESULTS.}

Another related problem is as follows; we know that the restriction of a Tauberian operator to any closed subspace is again Tauberian. So, 1s Theorem 1 true for Tauberian operators instead of semi-Fredholm operators?. The answer is obviously positive if, for instance $E$ is reflexive or $E$ is purely non-refelxive. However, we have,

THEOREM 3. Let $E$ be an infinite-dimensional Banach space which contains an infinite-dimensional somewhat reflexive closed subspace $M$ which is not reflexive. 
Then there exists an infinite-dimensional Banach space $F$ and a non-Tauberian surjective operator $\mathrm{T}: \mathrm{E} \longrightarrow \mathrm{F}$ such that every infintie-dimensional closed subspace $\mathrm{Z}$ of E contains an infinite-dimensional closed subspace $W$ for which $T / W$ is Tauberian.

PROOF. First assume that $E / M$ is infinite-dimensional and consider the quotient map $\mathrm{T}: \mathrm{E} \longrightarrow \mathrm{E} / \mathrm{M}$. It follows, as in the above example, that $\mathrm{T}$ is not Tauberian but that for every purely non-reflexive subspace $Z$ of $E$ then, $T \mid Z$ is Tauberian. Now, assume that $Z$ is not purely non-reflexive; in this case there exists an infinitedimensional reflexive subspace $W \subset Z$. For this $W$, it is obvious that $T \mid W$ is Tauberian.

If $\operatorname{dim} E / M<\infty$ then, $E$ is itself somewhat reflexive and non-reflexive. Since $E$ is not reflexive, there exists a bounded basic sequence $\left(e_{n}\right)$ in $E$ which is not weakly nul1 [8]. Without loss of generality, $\left(e_{2 n}\right)$ is not weakly null, otherwise use $\left(e_{2 n-1}\right)$. Let $N$ be the closed linear span of $\left(e_{2 n}\right)$. It follows that $N$ is a nonreflexive closed subspace of $E$ such that $E / N$ is infinite-dimensional. Let us prove that the quotient map $\mathrm{T}: \mathrm{E} \longrightarrow \mathrm{E} / \mathrm{N}$ satisfies the conclusion. Given an infinitedimensional closed subspace $Z$ of $E$ then, $Z$ contains an infinite-dimensional reflexive subspace $W$; it follows that $T \mid W$ is Tauberian. But, on the other hand, $T$ is not Tauberian because 1 ts null space $N$ is not reflexive.

ACKNOWLEDGEMENT. We are grateful to the referee for some valuable suggestions.

\section{REFERENCES}

1. NEIDINGER R., Properties of Tauberian Operators on Banach spaces, Ph. D. Dissertation, University of Texas at Austin, 1984.

2. GARLING D.J.H. and WILANSKY A., On a summability theorem of Berg, Crawford and Whitley, Proc. Cambridge Philos. Soc. 71(1972), 492-497.

3. KALTON N. and WILANSKY A., Tauberian operators on Banach spaces, Proc. Amer. Math Soc. 57(1976), 251-255.

4. NEIDINGER R. and ROSENTHAL H.P., Norm-attainment of linear functionals on subspaces and characterizations of Tauberian operators, Pacific J. of Math. $118(1985), 215-228$.

5. YANG K.M., The generalized Fredholm operators, Trans.Amer. Math. Soc. 216(1976), 313-326.

6. KATO T., Perturbation theory for nullity deficiency and other quantities of 1inear operators, J. Analyse Math. 6(1958), 273-322.

7. ROSENTHAL H.P., On totally incomparable Banach spaces, J. Funct. Ana1. 4(1969) $167-175$.

8. PELCZYNSKI A., A note on the paper of I. Singer "Basic sequences and reflexivity of Banach spaces", Studia Math. 21(1962), 371-374. 


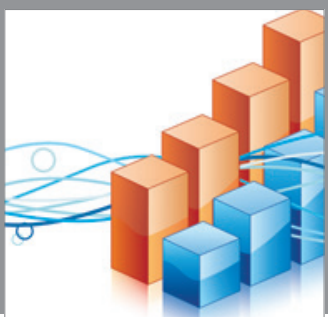

Advances in

Operations Research

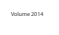

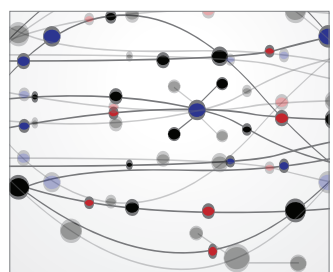

\section{The Scientific} World Journal
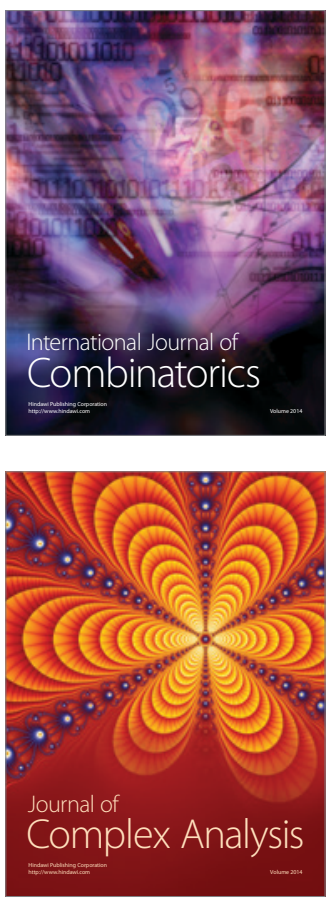

International Journal of

Mathematics and

Mathematical

Sciences
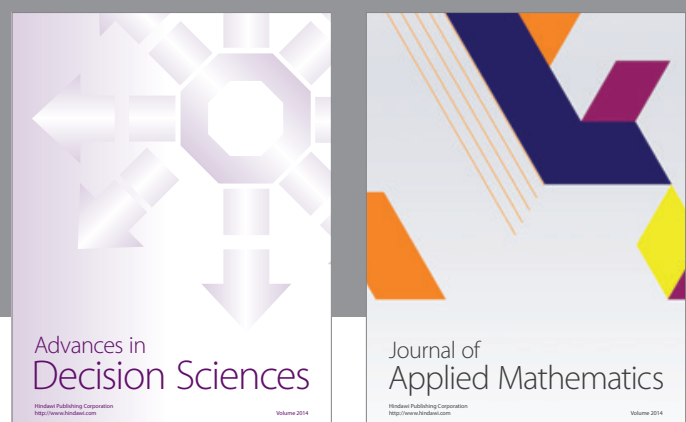

Journal of

Applied Mathematics
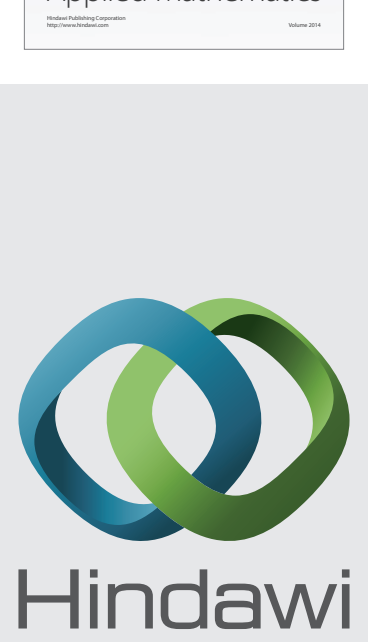

Submit your manuscripts at http://www.hindawi.com
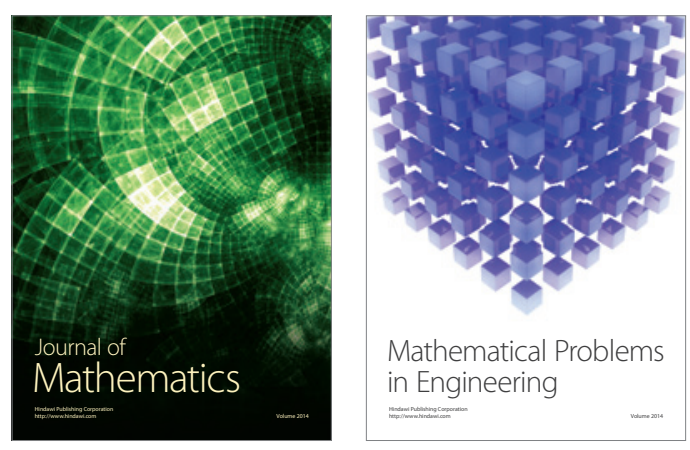

Mathematical Problems in Engineering
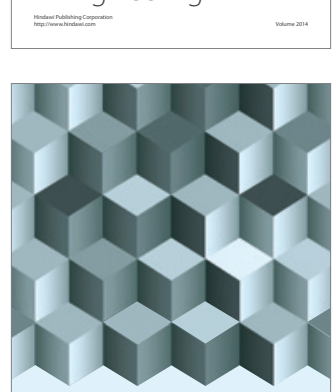

Journal of

Function Spaces
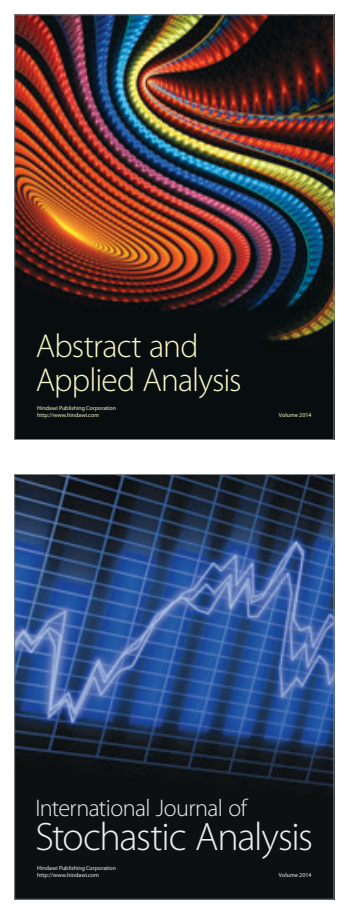

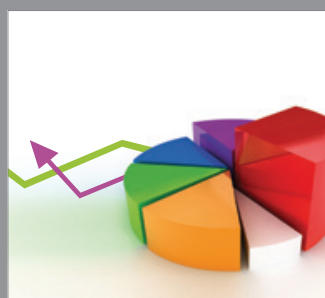

ournal of

Probability and Statistics

Promensencen
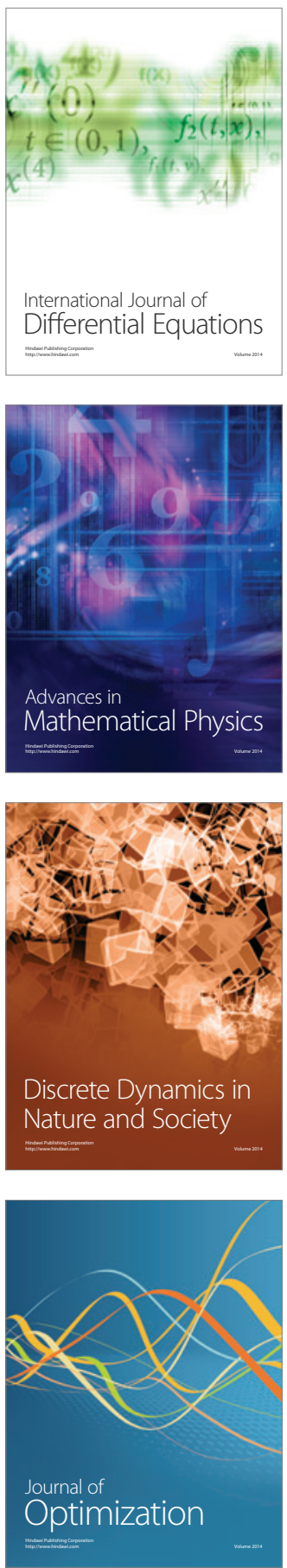